\title{
Panspermia and Horizontal Gene Transfer
}

\author{
Brig Klyce | Astrobiology Research Trust \\ 168 Grove Park Circle | Memphis TN 38117-3134 \\ http://www.panspermia.org/art.htm
}

\begin{abstract}
Evidence that extremophiles are hardy and ubiquitous is helping to make panspermia a respectable theory. But even if life on Earth originally came from space, biologists assume that the subsequent evolution of life is still governed by the darwinian paradigm. In this review we show how panspermia could amend darwinism and point to a cosmic source for, not only extremophiles but, all of life. This version of panspermia can be called "strong panspermia." To support this theory we will discuss recent evidence pertaining to horizontal gene transfer, viruses, genes apparently older than the Earthly evolution of the features they encode, and primate-specific genes without identifiable precursors.
\end{abstract}

Keywords: strong panspermia, horizontal gene transfer (HGT), viruses and evolution, old genes.

\section{THE SOFTWARE PROBLEM}

For life to originate in a lifeless environment, two kinds of problems must be solved. The first is the "hardware" problem - there must be nucleotides, proteins, and other components arranged in working order inside some kind of protective barrier. The second problem is the "software" problem - the genetic system needs the programming that directs development and all the other functions of life. Without software, the hardware is useless.

Unlike the hardware problem, the software problem does not go away after life first emerges on Earth. The programming that the simplest prokaryotic cells require for subsistence does not include programming for the complex features of higher life forms. Examples of these complex features include -

methanogenesis, photosynthesis, oxygen metabolism, nitrogen fixation, multicellularity, cell specialization, sexual reproduction, locomotion, digestive systems, circulatory systems, nervous systems, immune systems, leaves, shells, gills, lungs, limbs, bones, teeth, scales, skin, hair, wings, eyes, ears, etc.

All of these features are encoded by specific software. The huge and growing volume of genomic data enables us to identify the genes that code for components of many of these features. What the data do not show however, is clear evidence of how these genes "originated." As W. Ford Doolittle observed almost ten years ago, "Many eukaryotic genes ...seem to have come from nowhere." ${ }^{1}$ The intervening years have not changed this assessment.

\section{HGT AMONG PROKARYOTES}

There is a growing consensus that horizontal gene transfer (HGT) plays a major role in the evolution of prokaryotes. Not only single genes, but clusters and suites of genes, are swapped among prokaryotes like cassettes. Entire metabolic pathways, even if not clustered on a chromosome, are known to be installed by HGT $^{2,3}$. The transferred genes may help prokaryotes to metabolize different nutrients, or survive against new threats, but the types of transferred genes are not restricted. All types of genes are subject to transfer. 


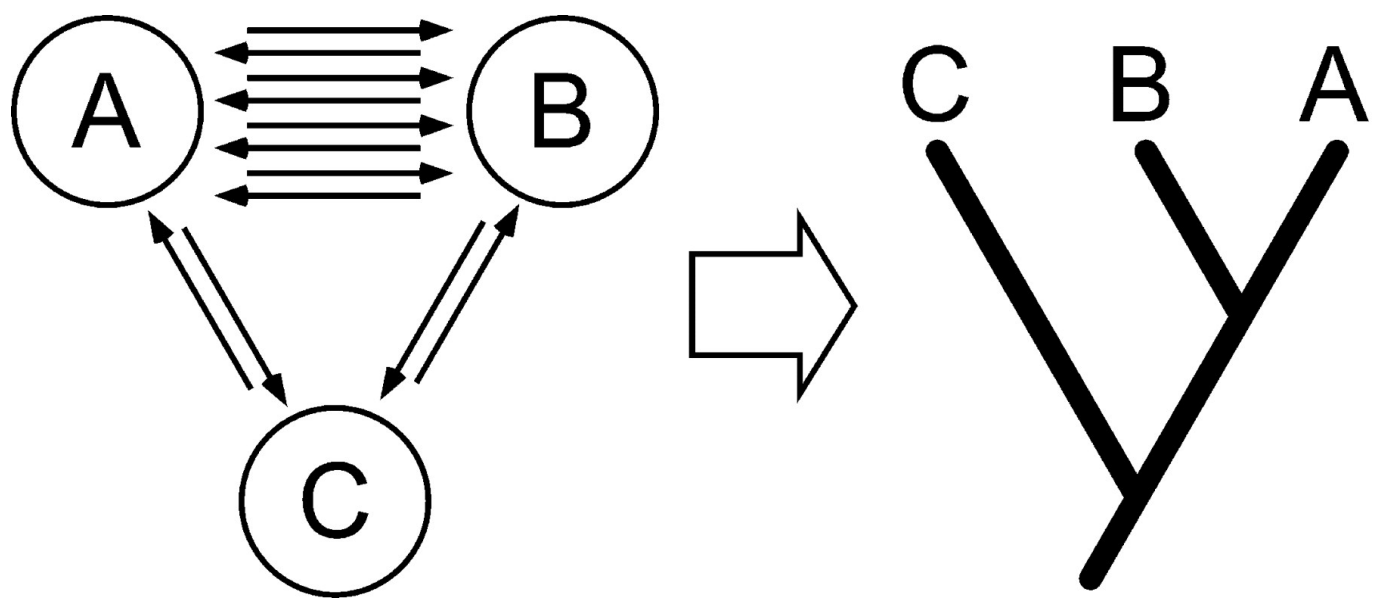

Figure 1. "Gene transfer can create patterns of similarity and difference that mimic patterns produced by vertical descent. If taxa A and B successfully exchange genetic information (by homologous recombination or HGT) more frequently with each other than with taxon $\mathrm{C}$, they will come to resemble each other more closely than they do C, both in gene content and gene sequence. Treelike patterns based on gene content or sequence will reflect these different frequencies, not some underlying organismal phylogeny...." (Gogarten et al.) ${ }^{4}$

The ubiquity of HGT among prokaryotes has even made us question the basic concept of "species" 5.

Ernst Mayr said that among prokaryotes, HGT is "all there is" ${ }^{6}$. The recognition of the importance of HGT among prokaryotes amounts to a complete, unnoticed paradigm shift.

\section{PHOTOSYNTHESIS BY GENE TRANSFER}

Among prokaryotes, a well-researched example of evolution by HGT is photosynthesis.

"The analysis revealed clear evidence that photosynthesis did not evolve through a linear path of steady change and growing complexity but through a merging of evolutionary lines that brought together independently evolving chemical systems - the swapping of blocks of genetic material among bacterial species known as horizontal gene transfer." ${ }^{7}$

In 2003 a team at the University of Warwick found that a bacteriophage that infects marine Synechococcus strains "encodes the D1 and D2 proteins of .... large protein-pigment complex that catalyses the lightdependent oxidation of water to molecular oxygen [in cyanobacteria]." 8

Later, a team of biologists from Boston and San Diego "report the presence of genes central to oxygenic photosynthesis in the genomes of three phages from two [other] viral families...." ${ }^{9}$

\section{HGT AMONG EUKARYOTES}

Eukaryotes have even more ways than prokaryotes to receive transferred genes. The most obvious example of HGT among eukaryotes is endosymbiosis - a whole prokaryote is engulfed into a eukaryotic cell as an organelle. The organelle may then transfer genes from itself to the eukaryotic nucleus. This is a wholesale method of HGT that is no longer controversial. The phenomenon is especially well-documented in Acquiring Genomes, by Margulis and Sagan. ${ }^{10}$

Similar gene transfer can also occur between a eukaryotic host and its extracellular parasites, such as our own e. coli. Eukaryotes can even acquire genes from their food.

And viruses can install genes into eukaryotes. We used to think that the germ line of 
multicelled eukaryotes was protected from these viruses, but this is wrong. In fact, $6-8 \%$ of the human genome consists of recognizable endogenous retroviruses (ERVs) ${ }^{11}$. This discovery prompted John Coffin to suggest that we have more viruses than genes in our genes!

"Endogenous proviruses are widespread in nature, and have been found in all vertebrate and most invertebrate species that have been studied, indicative of the barrage of retroviruses to which all species have been subjected throughout their evolutionary history. In humans, there are about 80,000 proviruses or their remnants, comprising about $6-8 \%$ of the genome, or about twice as many as genes. In other words, there are more proviruses in us than there is us in us." 12

Most of these ERVs apparently entered the human lineage between 10 and 50 million years ago, but many more ERVs may also be present and no longer recognizable as such.

\title{
5. THE IMMUNE SYSTEM BY GENE TRANSFER
}

Among Eukaryotes, the situation is reminiscent of that for prokaryotes a decade ago - examples of evolution by HGT are already too numerous to keep count. One of the most salient examples is the immune system of jawed vertebrates. In 1998, a team at Yale wrote, "We owe the repertoire of our immune system to one transposon insertion, which occurred 450 million years ago in the ancestor of the jawed fishes." 13

Seven years later, a pair at the Genetic Information Research Institute found, "the approximately 600amino acid 'core' region of RAG1 required for its catalytic activity is significantly similar to the transposase encoded by DNA transposons that belong to the Transib superfamily [found in the genomes of]...the fruit fly and African malaria mosquito..., sea urchin, yellow fever mosquito, silkworm, dog hookworm, hydra, and soybean rust" ${ }^{14}$. It is noteworthy that many of the carriers of this genetic program are infectious agents.

This evidence was cited in the trial in Dover PA in December 2005 (Kitzmiller et al. v. Dover Area School District) to support the Darwinian theory of evolution - but it does not support Darwinism. How a sequence of some 1,800 nucleotides could acquire programming for a function not deployed by the species containing it is not explained by Darwinism. Instead it is a jarring puzzle.

\section{VIRUSES WELL SUITED TO TRANSFER GENES}

\begin{abstract}
"Because there are an estimated $10^{31}$ phages on the planet and they can move between environments, the potential reservoir of genes that can be transferred both locally and globally by phage is enormous. ...There is little restriction to the types of genes carried by the viral community, suggesting that they influence a wide range of processes, including biogeochemical cycling, short-term adaptation and longterm evolution of microbes." 15
\end{abstract}

Most phages are small, but some eukaryotic viruses, notably the recently discovered mimivirus, are large enough to contain hundreds of genes. A recent study of the mimivirus speculates that its unique vertex may function as a genome delivery port ${ }^{16}$. Because of their infectivity, viruses can transform whole populations in a single generation, as Susumu Ohno ${ }^{17}$ wrote, in 1970.

\section{GENES FOR NERVOUS SYSTEMS OLDER THAN NERVOUS SYSTEMS}

If HGT is the primary driver of evolution, then the genes to be transferred must already exist, before they are installed. If so, there should be evidence for genes that exist before they are deployed. There is such evidence. A good example is the genes for nervous systems. Genes that code for many components of nervous systems in higher animals are found in more primitive species that lack nervous systems. In 2008, these were observed in Trichoplax ${ }^{18}$, and sponges ${ }^{19}$. 
The genome of the placozoan Trichoplax adhaerens indeed delivers some notable evidence that the genetic inventory may precede morphological manifestation of organs. For example, the placozoan genome harbors representatives of all major genes that are involved in neurogenesis in higher animals, whereas placozoans show not the slightest morphological hint of nerve or sensory cells. ${ }^{20}$

Evidence like this prompted Casey Dunn of Brown University to comment, "It is now completely clear that genomic complexity was present very early on." 21

\section{PRIMATE-SPECIFIC GENES LACK PRECURSORS}

An international team identified 131 transcriptional units (TUs) in the human genome that are found only in other primates. These 131 genes lack identifiable precursors in any sequenced non-primate genomes. This lack did not improve even after the criteria for identifying homologs was relaxed. The team concluded, "Primate-specific TUs are consistent with de novo insertions, not sequence divergence." ${ }^{22}$

This finding is surprising for darwinian theory because, if the genes arose by darwinian trial-and-error, there would be precursors. But the finding is consistent with our prediction, "If a new genetic program arrives by the strong panspermia process, intervening species should possess either nearly identical versions of it... or nothing similar...." 23

\section{SUMMARY}

HGT is important because it means that all of life, not just the first life on Earth, could come from space. Of course this version, "strong" panspermia, is speculative. And it is subject to the same objection that is made about basic panspermia - it simply removes the origin of genetic programs to some other venue. So why should we even consider the possibility that HGT drives evolutionary advances? Because the weight of evidence points to it! The evidence now includes -

A complete paradigm shift to HGT in our concept of prokaryotic evolution Hundreds of important examples of innovation by HGT in eukaryotes Clear evidence that viruses install genes among all forms of life The existence of genes before the evolution on Earth of the features they encode. The discovery of primate-specific genes without precursors.

\section{REFERENCES}

[1] W. Ford Doolittle, Sci. Am. 90, 282 (2000).

[2] Maslov, S. et al., "Toolbox model of evolution of prokaryotic metabolic networks and their regulation," Proc. Natl Acad. Sci. USA, online 29 May (2009).

[3] Iwasaki, W. and Takagi, T. "Rapid Pathway Evolution Facilitated by Horizontal Gene Transfers across Prokaryotic Lineages," PLoS Genetics, 5(3): e1000402 (2009).

[4] Gogarten, J.P., Doolittle, W.F. and Lawrence, J.G., "Prokaryotic Evolution in Light of Gene Transfer," Mol. Biol. \& Evol., 19, 2226-2238 (2002).

[5] Goldenfeld, N. and Woese, C., "Biology's next revolution," Nature 445, 369 (2007).

[6] Mayr, E., interviewed by Brig Klyce, Cambridge MA, 7 Jun 2000.

[7] Raymond, J. et al., "Whole-Genome Analysis of Photosynthetic Prokaryotes," Science 298, 1616-1620 (2002). 
[8] Mann, N.H. et al., "Marine ecosystems: Bacterial photosynthesis genes in a virus," Nature 424, 741 (2003).

[9] Lindell, D. et al., "Genome-wide expression dynamics of a marine virus and host reveal features of coevolution," Nature 449, 83-86 (2007).

[10] Margulis, L. and Sagan, D., [Acquiring Genomes: A Theory of the Origins of Species], Basic Books, (2002).

[11] Hughes, J. F. and Coffin, J.M., "Evidence for genomic rearrangements mediated by human endogenous retroviruses during primate evolution," Nature Genetics 29, 487-489 (2001).

[12] Coffin, J.M., "Evolution of Retroviruses: Fossils in our DNA," Proc. Am. Phil. Soc., 148(3) 264-280 (2004).

[13] Agrawal, A., Eastman, Q.M. and Schatz, D.G. "Transposition mediated by RAG1 and RAG2 and its implications for the evolution of the immune system," Nature 394, 744-751 (1998).

[14] Kapitonov, V.V, and Jurka, J., "RAG1 Core and V(D)J Recombination Signal Sequences Were Derived from Transib Transposons," PLoS Biology 3(6), e181(2005).

[15] Dinsdale, E.A. et al., "Functional metagenomic profiling of nine biomes," Nature 452, 629-632 (2008).

[16] Xiao, C. Kuznetsov, Y.G, Sun, S., Hafenstein, S.L., Kostyuchenko, V.A, et al., "Structural Studies of the Giant Mimivirus," PLoS Biology 7(4), e92 (2009).

[17] Ohno, S. [Evolution by Gene Duplication], Springer-Verlag (1970).

[18] Srivastava, M. et al., "The Trichoplax genome and the nature of placozoans," Nature 454, 955-960 (2008).

[19] Richards, G.S., Simionato, E. et al., "Sponge Genes Provide New Insight into the Evolutionary Origin of the Neurogenic Circuit," Current Biology 18, 1156-1161 (2008).

[20] Schierwater B, Eitel M, Jakob W, Osigus HJ, Hadrys H, et al., "Concatenated Analysis Sheds Light on Early Metazoan Evolution and Fuels a Modern 'Urmetazoon' Hypothesis," PLoS Biology 7(1), e20, (2009).

[21] Pennisi, E. "'Simple' Animal's Genome Proves Unexpectedly Complex," Science 321, 1028-1029 (2008).

[22] Tay, S-K, Blythe, J and Lipovich, L. "Global discovery of primate-specific genes in the human genome," Proc. Natl Acad. Sci. USA 106, 12019-12024 (2009).

[23] Ray, T and Klyce, B, "New genetic programs in Darwinism and strong panspermia," http://www.panspermia.org/graphspaper.htm (2002). 